\author{
Repetitive nasal allergen challenge in allergic rhinitis: priming and Th2-type \\ inflammation but no evidence of remodelling \\ Nara Orban, Mikila R Jacobson, Kayhan T Nouri-Aria, Stephen R Durham, Aarif O Eifan
}

Running Title: Repetitive nasal allergen challenge in allergic rhinitis.

Total word count: 2996

Table count: 2

Figure count: 3

\begin{abstract}
Affiliations: Allergy and Clinical Immunology, National Heart and Lung Institute, Medical Research Council and Asthma UK Centre for Allergic Mechanisms of Asthma, Faculty of Medicine, Imperial College London, London, United Kingdom
\end{abstract}

Address for Correspondence:

Dr Aarif O. Eifan

Allergy and Clinical Immunology

Royal Brompton Hospital, NHLI,

Imperial College London,

Dovehouse St, London, SW3 6LY

e.mail: a.eifan@imperial.ac.uk

Author has contributed in the hypothesis's delineation and design of the study. Involved in acquisition, analysis and interpretation of the data. Wrote the manuscript and revised the article prior to submission. 
Nara Orban, Mb B Chir FRCS: Allergy and Clinical Immunology, National Heart and Lung Institute, Imperial College London. nara.orban@nhs.net. Author has contributed in the hypothesis's delineation and design of the study. Involved in acquisition, analysis and interpretation of the data with involvement in article revision prior to submission.

Mikila Jacobson, PhD: Allergy and Clinical Immunology, National Heart and Lung Institute, Imperial College London.m.jacobson@imperial.ac.uk. Author has contributed in acquisition of the data and the analysis and interpretation of data with involvement in article revision prior to submission.

Kayhan Nouri-Aria, PhD: Allergy and Clinical Immunology, National Heart and Lung Institute, Imperial College London. kayhan.baber@btinternet.com. Author has contributed in acquisition of the data and the analysis and interpretation of data with involvement in article revision prior to submission.

Stephen R Durham, MD FRCP: Allergy and Clinical Immunology, National Heart and Lung Institute, Imperial College London. s.durham@imperial.ac.uk. Author has contributed in the conception, hypotheses delineation, and design of the study. Interpretation of data and reviewed final manuscript before submission.

Disclosure of conflict of interest: Authors declare no conflict of interests.

Support: The study was funded by the Academic Drug Discovery Initiative between Imperial College Trust and GlaxoSmithKline. 


\section{Abstract \\ Repetitive nasal allergen challenge in allergic rhinitis: priming and Th2-type inflammation but no evidence of remodelling} \\ Nara Orban, Mikila R Jacobson, Kayhan T Nouri-Aria, Stephen R Durham, Aarif O Eifan
}

Background: Local tissue eosinophilia and Th2-cytokines are characteristic features of seasonal allergic rhinitis. Airway-remodelling is a feature of asthma whereas evidence for remodelling in allergic rhinitis (AR) is conflicting.

Objective: By use of a novel human repetitive nasal allergen challenge (RAC) model, we evaluated the relationship between allergic inflammation and features of remodelling in AR.

Methods: Twelve patients with moderate-severe AR underwent 5-alternate day challenges with diluent which after 4-weeks were followed by 5-alternate day challenges with grass pollen extract. Nasal symptoms, Th1/Th2 cytokines in nasal secretion and serum were evaluated. Nasal biopsies were taken 24 hours after the $1^{\text {st }}$ and $5^{\text {th }}$ challenges with diluent and with allergen. Sixteen healthy controls underwent a single challenge with diluent and with allergen. Using immunohistochemistry, epithelial and sub-mucosal inflammatory cells, and remodelling markers were evaluated by computed image analysis.

Results: There was an increase in early and late-phase symptoms after every allergen challenge compared to diluent (both $\mathrm{p}<0.05$ ) with evidence of both clinical and immunological priming. Nasal tissue eosinophils and IL-5 in nasal secretion increased significantly after RAC compared to corresponding diluent challenges ( $\mathrm{p}<0.01, \mathrm{p}=0.01$, respectively). There was a correlation between submucosal mast cells and the early-phase clinical response ( $r=0.79$, $\mathrm{p}=0.007$ ) and an association between epithelial eosinophils and IL-5 concentrations in nasal secretion $(r=0.69, p=0.06)$ in allergic rhinitis. No differences were observed after RAC with regards to epithelial integrity, reticular basement membrane thickness, glandular area, expression of markers of activation of airway-remodelling including $\alpha$-SMA, HSP-47, extracellular matrix (MMP7, 9 and TIMP-1), angiogenesis and lymphangiogenesis for AR compared to healthy controls.

Conclusion: Novel repetitive nasal allergen challenge in participants with severe persistent seasonal allergic rhinitis resulted in tissue eosinophilia and increases in IL-5 but no structural 
changes. Our data support no link between robust Th2-inflammation and development of 83 airway-remodelling in AR.

84 Key words: Allergic, rhinitis, remodelling, early and late phase response, priming,

85 inflammation, eosinophils, mast cells, lymphangiogenesis, angiogenesis, repetitive 86 allergen challenge

87 Abstract WORD: 292

88 Abbreviation

89 AR: Allergic rhinitis

90 AUC: Area under the curve

91 EPR: Early Phase Response

92 HC: Healthy controls

93 LPR: Late phase Response

94 RAC: Repetitive Allergen Challenge

95 NAC: Nasal allergen challenge

96 TNSS: Total nasal symptom scores

97

98

99 
Allergic rhinitis and asthma are widely accepted to be Th2-type airway inflammatory diseases in which production of allergen-specific IgE, accumulation of eosinophils, mast cells and increases in Th2 cytokines are linked to pathophysiology. ${ }^{1-3}$

In allergic asthma, repeated exposure to airborne allergens is known to result in persistent inflammation, airway epithelial damage and remodelling. In contrast, whether remodelling occurs in allergic rhinitis remains controversial. ${ }^{4}$ A recent statement from the American Thoracic Society reports a lack of consensus regarding importance of multiple features of airway remodelling. ${ }^{5}$ Understanding the mechanisms of airway remodelling and their impact on airway disease, may inform novel therapeutic approaches.

111 Nasal allergen challenge allows precise study of the time course of clinical, cellular and immune responses following therapeutic interventions with the advantage of small sample sizes. ${ }^{6}$ Repetitive inhaled allergen challenge in asthma has been used to imitate natural allergen exposure whilst being tailored to induce detectable Th2 inflammation and eosinophilia without inducing severe asthma symptoms, hence being used in both translational research and clinical settings. ${ }^{7-9 .}$

Nasal allergen provocation in murine models has contributed significantly to our knowledge of pathophysiology and has often been developed with chronic repetitive challenges. ${ }^{10}$ It has been questioned whether nasal allergen challenge in a human setting adequately reflects natural environmental exposure to allergens. Hence, longer periods of repetitive allergen challenge and appropriate controls need to be validated in humans.

The purpose of this study in participants with severe persistent seasonal allergic rhinitis was to investigate the effect of chronic repetitive nasal grass pollen allergen challenge ( 5 challenges 
125 and priming with a detailed evaluation of evidence for structural remodelling in the nasal 126 mucosa.

127

128

129

130

131

132

133

134

135

136

137

138

139

140 


\section{Study Subjects (Please see supplement)}

Subjects aged 18-55 years with clinical history of moderate-severe persistent allergic rhinitis according to ARIA guideline ${ }^{11}$ (please see supplement). The study was approved by the National Ethics committee (ref. 07/Q0404/64) and was performed with the subjects' informed written consent.

\section{Study design}

Twelve allergic rhinitis participants and 16 non- allergic healthy controls were studied (Table 1). During the trial period non-allergic healthy participants attended the clinic four times out of season. Each participant underwent a diluent challenge followed 4 weeks later by an allergen challenge. Twenty-four hours after each challenge, participants had a biopsy of their inferior turbinate. During the trial period allergic rhinitis participants attended the clinic 14 times over the course of 8-10 weeks as shown in Fig 1. Allergic rhinitis participants had 7 visits out of pollen season for the diluent phase followed by 7 visits for the allergen challenge phase of the study. For each phase, 5 visits were for the challenge (diluent or allergen) and 2 were for nasal biopsy. Participants completed the symptom score questionnaires at regular intervals for 24 hours prior to and following each challenge. The biopsies took place 24 hours after the first and the last challenge in both phases. Each side of the nose was randomly designated as either side 1 or side 2 . This was determined on the first diluent challenge visit by 'flip of a coin' for both allergic and healthy participants. Both sides of the nose were challenged on each challenge visit but only one side of the nose was biopsied. Blood samples and nasal secretions were taken to determine markers of the allergic response immediately prior to the nasal biopsies. 
165 Skin prick testing was performed as previously described. ${ }^{12}$

\section{Repetitive nasal allergen challenge (RAC), symptoms scores and nasal secretion}

RAC and nasal symptoms scores

Nasal provocation was performed using Aquagen-225 diluent for control challenges and

Timothy grass pollen freeze-dried extract, Phleum pratense, reconstituted in diluent (Aquagen spray (Bidose; Valois S.A., Marly le Roi, France), 500 biologic units (BU)/100 $\mu$ L was administered as a single dose to each nostril. ${ }^{13}$ Symptoms were evaluated on a four-point scale: 0 (no symptoms) to 3 (severe symptoms) for sneezing, nasal discharge, itching and nasal obstruction (Total score range 0-12). Total nasal symptom scores were recorded at screening, before challenge and at 5 minutes, 10 minutes, 30 minutes and 1-24 hours after each RAC reactions-TNSS (LPR-TNSS) area under the curve (AUC).

Nasal secretion procedure, measurements of nasal secretion and serum cytokine levels (please see supplement).

\section{Nasal Biopsies, immunohistochemistry staining and quantification}

Nasal biopsies $(2.5 \mathrm{~mm})$ were taken from the under surface of the inferior turbinate. Immunohistochemistry was performed using the avidin/biotin complex-alkaline phosphatase (ABC-AP) method as previously described ${ }^{12,14}$ (Please see supplement). 


\section{Statistics}

189 Values are presented as median [IQ 25-75 range] unless otherwise specified. Comparisons for 190 quantitative variables were performed by non-parametrical analysis; Wilcoxon matched-pairs 191 signed-rank test for within group comparisons. Furthermore, remodelling markers were 192 reported as a delta outcome (change from diluent) and comparisons were performed by nonparametrical analysis; Mann-Whitney $\mathrm{U}$ test for between groups. The AUC is calculated by taking the per-average of the TNSS AUC measured at 0 to 1 hours (EPR) and the per-hour average of the AUC measured between 1 to 10 hours (LPR). Spearman correlation coefficients were used to assess the relationships between symptoms and biomarkers. Inter-observer reproducibility within 2 standard deviation was measured using Bland-Altman test. All tests were two-tailed. Significance was set at $\mathrm{P}<0.05$. GraphPad Prism 6 Project software was used 199 for analysis. 


\section{Results}

208

209

210

211

212

213

214

215

216

217

\section{Subjects}

The demographic characteristics are summarised in Table 1. Twenty-eight subjects (12 AR [F:M; 4:8, median age 27] and 16 normal controls [F:M; 10:6, median age 29]) were included for repetitive grass pollen allergen challenge study. Two (16\%) AR subjects were sensitized to perennial allergens without symptoms. Eight percent of AR patients also had previous history of eczema and $16 \%$ had mild seasonal asthma (Table 1).

\section{Symptoms after repetitive nasal allergen challenge}

Participants with seasonal allergic rhinitis had bothersome seasonal total symptoms scores compared to healthy control (Median [IQ]; 7 [4-9] vs 0, p<0.0001, Table 1). Response to repetitive allergen challenge was assessed by monitoring the early phase-TNSS (EPR-TNSS, 0-1 hour) and late phase reactions-TNSS (LPR-TNSS, 1-24 hours).

\section{Early phase symptoms (TNSS 0-1hr):}

The early phase TNSS increased significantly in repetitive allergen challenge group after each challenge when compared to the corresponding diluent challenge in the same participants for each of the 5 paired repetitive challenges ( $p<0.05$, respectively) throughout 11 days. The early phase TNSS increased gradually from the initial allergen challenge reaching a peak after the third challenge ( $\mathrm{p}=0.01$, median [IQ]; 4.7 [3-6] vs 3.5 [2-5]), and then remained consistently high for the final 2 provocation tests compared to control challenges (Fig. 2A). Control participants showed minimal increases in their early phase TNSS after the initial control challenge but no change after allergen challenge $(\mathrm{p}=0.03$, median [IQ]; 0.7 [0.5-0.8] vs 0 [00.2]) (Fig. 2 A), likely related to a minor irritant effect of the procedure with adaptation on rechallenge. 
Late phase symptoms (TNSS 1-24hr): A significant increase in late-phase TNSS was observed after all 5 repeated allergen doses when compared to corresponding diluent challenges at each time point. The magnitude of LPR-TNSS also increased gradually from the initial allergen challenge progressively over the 5 challenges and was significantly higher after the $5^{\text {th }}$ allergen challenge compared to the first and third challenges ( $\mathrm{p}=0.01$ and $\mathrm{p}=0.02$, respectively). Normal control participants demonstrated no changes in LPR-TNSS after allergen when compared to diluent (Fig. 2 B, eFig 1 A-B).

Eosinophils: Total nasal tissue eosinophils increased after both the first and fifth allergen challenge compared to corresponding diluent challenges $(\mathrm{p}=0.01$ and $\mathrm{p}<0.01$, respectively) (Fig 3A, Table 2). This increase was mainly due to increases in submucosal tissue eosinophils $(\mathrm{p}<0.01$ and $\mathrm{p}=0.03$, respectively) (Table 2), with a trend for an increase in epithelial eosinophil counts ( $\mathrm{p}=0.06)$ after the fifth allergen challenge but not for the first challenge when compared to corresponding diluent. No changes in nasal eosinophils were observed in healthy controls (Table 2).

Mast cells and basophils: In participants with allergic rhinitis there were no changes in the numbers of mast cells or basophils after either the $1^{\text {st }}$ or $5^{\text {th }}$ nasal allergen challenge when compared to the corresponding diluent challenge or with responses in normal control participants (Table 2). However, a highly significant correlation was observed between the early-phase (0-60 min) TNSS after the first allergen challenge and the number of nasal submucosal mast cells $(r=0.79, \mathrm{p}=0.007)($ Fig $2 \mathrm{C})$.

\section{Cytokines in nasal secretion and serum cytokines}

Nasal cytokines: In allergic participants, IL-5 concentrations were markedly and consistently increased after the $5^{\text {th }}$ repeated allergen challenge (day 11) compared to diluent challenge $\left(p=0.01\right.$, Fig 3B). There was a trend for the increased nasal levels of IL-5 after the $5^{\text {th }}$ challenge 
to correlate with the corresponding nasal epithelial eosinophil counts ( $r=0.62, p=0.06$; Fig 3C).

255

256

257

In contrast, nasal IL-4 concentrations were low, at or below the limit of detection for the assay and no significant alterations were observed after all 5 allergen challenges for the allergic groups. There were no significant changes in concentration of IL-10, IL-13 or IFN- $\gamma$ after the last $\left(5^{\text {th }}\right)$ dose of repetitive allergen challenge compared to its diluent. After the initial dose of allergen challenge in allergic participants there were no significant changes observed in all nasal cytokines when compared to their diluent. Likewise, no changes in levels of nasal cytokines were detected in healthy controls after allergen challenge compared to diluent (Table 2).

Serum cytokines: No changes in serum concentrations of IL-4, IL-5, IL-10 nor IFN- $\gamma$ were observed after allergen challenges within allergic participants when compared to diluent challenges (Table 2). There were no changes in concentration of serum IL-13 after the last (5th) dose of repetitive allergen challenge compared to its diluent. In normal control subjects there was a small reduction in serum IL-4 concentration $(p=0.04)$ after allergen compared with diluent, otherwise no changes in levels of serum IL-5, IL-10 nor IFN- $\gamma$ cytokines were detected in healthy controls after allergen challenge compared to diluent (Table 2).

\section{Effect of repetitive allergen challenge on upper airway remodelling}

No significant changes in epithelial integrity, reticular basement membrane thickness nor glandular area (epithelial mucus content measured by epithelial area occupied by goblet cells $(\%)$, and mucus (\%) staining area in submucosa) were detected in allergic participants after either the $1^{\text {st }}$ or $5^{\text {th }}$ allergen challenges compared to healthy controls (eTable 1). Similarly, no differences in expression of markers of activation of airway-remodelling including $\alpha$-SMA, HSP-47 and extracellular matrix (MMP7, 9 and TIMP-1) nor angiogenesis or 
277 lymphangiogenesis were observed for either AR or healthy controls after allergen challenge

278 compared to corresponding diluent, with no differences between groups (eTable 1).

279

280

281

282

283

284

285

286

287

288

289

290

291

292

293 
Repetitive grass pollen allergen challenge performed out of season in participants with moderate-severe allergic rhinitis resulted in typical immediate and late-phase responses. Clear evidence of priming was observed with alternate-day allergen challenges resulting in increases in the magnitude of the immediate $(0-60 \mathrm{~min})$ nasal response that peaked at the $3^{\text {rd }}$ challenge performed after 5 days compared to the first challenge and in the magnitude of the late-phase response (1-24 hours) that peaked at the $5^{\text {th }}$ challenge after 11 days. The number of mucosal mast cells correlated with the magnitude of the IgE-dependent early-phase nasal symptom response. Allergen-induced late-phase nasal symptoms were accompanied by increases in the Th2 cytokine IL-5 in nasal secretion that correlated with parallel increases in the number of nasal mucosal eosinophils on day 11. Despite these clear symptomatic responses with nasal priming and Type 2 inflammation, no significant alterations in markers of remodelling, including epithelial integrity, mucus glands, blood vessel and lymphatic vascular changes nor to anti-IgE or targeting Th2 cytokines, therapeutic strategies against remodelling in severe allergic rhinitis are unlikely to be successful.

In order to eliminate confounding factors such as use of anti-inflammatory therapies and variability of allergen concentration during natural exposure, allergen challenges were performed outside the pollen season when patients were asymptomatic and off all anti-allergic medication. The repetitive allergen challenge procedure and associated repetitive sampling of nasal fluid and repeat nasal biopsies in the same subjects was safe and well-tolerated. A further strength of the study was the inclusion of well-selected severe allergic rhinitis patients, longitudinal comparisons, the control of each of 5 repetitive challenges with 5 temporally 
identical control diluent challenges in the same individuals, the inclusion of sampling of the mucosa after a first single allergen as well as repetitive 5 alternate day challenges in the same individuals and the inclusion of a washout period of at least 4 weeks between the sequences of diluent and allergen challenges in order to avoid carry-over effects. Finally, healthy subjects were also used as negative control participants in order to control for the effects of allergen in the absence of atopic status and to control for effects of the clinical procedures and/or any temporal changes independent of the clinical protocol.

Previously, several studies have been conducted on the effect of repeated nasal allergen challenge and its relation with early and late allergic responses, dynamics of inflammatory cells and treatment effects. ${ }^{15-18}$ Studies in rhinitis have mainly detected the EPR after allergen challenge with only few reports of the LPR. ${ }^{19-22}$ The nasal LPR is less distinct post-nasal challenge than the late phase bronchial response in asthmatics after inhaled allergen challenge. ${ }^{23}$ The dominant nasal late nasal symptom that persists is nasal blockage and to a lesser extent rhinorrhoea. This variability of detecting clear-cut late nasal responses in studies involving either single or repetitive allergen challenge may be attributable to factors such as the lack of dynamic change in the nose, the duration of repetitive allergen challenge and the duration of symptom assessment after allergen challenge that varies from a few hours up to 24 or 48 hours. ${ }^{6}$ In this study in which nasal symptom scores were collected for 24 hours, more than $50 \%$ of participants developed a late-phase nasal response. There was no accumulation of local nasal basophils but a strong correlation between numbers of mast cells and early phase response was observed indicating the importance of mast cell activity during the early phase reaction and corroborating previous findings. Despite this correlation there was no significant difference observed when mast cell numbers were compared following allergen and diluent challenge. There was a significant increase in magnitude of LPR after the third dose of repeated allergen challenge (6 days after an initial challenge) with an increase in local late phase reaction 
inflammatory responses eosinophil and IL-5 expression which is in line with previous findings. ${ }^{24}$

345 As with previous reports that repetitive allergen challenge induces priming, ${ }^{25-27}$ our model produced significant increases in the magnitude of nasal symptoms after the third dose of repeated allergen challenge, six days after an initial challenge. Priming phenomenon was evidenced by an increase in tissue eosinophils and IL5 cytokines in nasal secretion after the fifth repetitive allergen challenge. Interestingly, there was a correlation between eosinophils migrating into the epithelium and IL5 in nasal secretions after persistent exposure allergen challenge. The current study provides insight into the priming phenomenon confirmed by clinical symptoms coinciding with physiological/immunological changes at the tissue level. Other studies have shown that the nasal priming phenomenon appears to be sensitive to topical corticosteroids that likely act by suppressing local Th2 cytokines release and associated tissue eosinophilia. $^{28,29}$

In contrast to allergic rhinitis, single allergen inhalation challenge in asthma has been shown to increase lower airway remodelling. ${ }^{30,31}$ In one study, methacholine inhalation challenge induced late features of remodelling, suggesting that mechanical factors accompanying bronchoconstriction per se and independent of allergen may provoke remodelling of the lower airways in asthma. As there is a large variability of individual response to allergen during natural allergen exposure, it might be difficult to assess the effect of inflammation on the local target organ. We used our novel human-nasal repetitive allergen challenge model out of natural pollen season to evaluate the relationship between chronic local Th2 inflammation and tissue remodelling.

Several repetitive chronic challenge models of asthma have been shown to induce local IL5 and eosinophilia in both mice and humans with both being associated with remodelling ${ }^{32-34}$ in 
some, but not all studies, ${ }^{35-37}$ and recent murine studies using eosinophil deficient mice and a transgenic mouse model of chronic Th2 driven inflammation with overexpression of IL-5 and eotaxin have shown that eosinophil degranulation and IL-5 may not be a driving source of pulmonary damage and/or remodelling. ${ }^{38,}{ }^{39}$ Furthermore, trials using anti-IgE, anti-IL5 and anti-CCR3 to target eosinophils and Th2 inflammation in animal models were promising, but clinical trials have only demonstrated efficacy on asthma symptoms in subsets of asthmatics but not remodelling... ${ }^{40-42}$

In the present study, the close correlation between mast cell numbers and the magnitude of the early response is consistent with the known IgE-mast cell associated dependency of the immediate allergic response. The increases in local IL-5 after the fifth nasal allergen challenge that paralleled nasal mucosal eosinophilia (but no increase in basophils) highlights the role of Th2-cytokines and eosinophils in the genesis of late-phase responses. However, in contrast to the lower airways, even repeated nasal challenge with repeated late responses and persistent chronic type 2 inflammation was insufficient to provoke features of upper airway remodelling in allergic rhinitis as observed in asthma. These findings are consistent with those of a recent study of the upper airway performed during natural allergen exposure in patients with moderate-severe persistent allergic rhinitis ${ }^{12}$. It was shown that despite the presence of severe disease and persistent upper airway allergic inflammation there was no evidence of nasal structural remodelling. These results might support the notion that either the upper airways respond differently to allergic inflammation than the lower airways or there are factors other than eosinophils or Th2 cytokines that contribute to tissue remodelling in lower airways.

In conclusion, the present study demonstrated that repetitive allergen challenge in humans induces early and late phase allergic response and priming. Moreover, a marked Th-2 mediated inflammation involving Th2 cytokine IL-5 and eosinophils was demonstrated. This study 
391

392

393

394

395

396

397

398

399

401

402

403

404

405

406

407

408

409

98

\section{6}

\section{7}

400

confirms that there is a strong association between Th2 inflammation and persistent allergic responsiveness but not structural nasal airway remodelling.

\section{3}

94

\section{5}

6

97

99


1) Bousquet J, Mantzouranis E, Cruz AA, et al. Uniform definition of asthma severity, control, and exacerbations: document presented for the World Health Organization Consultation on Severe Asthma. J Allergy Clin Immunol 2010;126:926-38.

2) Wheatley LM, Togias A. Clinical practice. Allergic rhinitis. N Engl J Med. 2015 Jan 29;372(5):456-63.

3) Lloyd CM, Hessel EM. Functions of T cells in asthma: more than just $\mathrm{T}(\mathrm{H}) 2$ cells. Nat Rev Immunol 2010;10:838-48.

4) Bousquet J, Jacot W, Vignola AM, Bachert C, Van Cauwenberge P. Allergic rhinitis: a disease remodeling the upper airways? J Allergy Clin Immunol 2004;113:43-49.

5) Prakash YS, Halayko AJ, Gosens R, et al. An Official American Thoracic Society Research Statement: Current Challenges Facing Research and Therapeutic Advances in Airway Remodeling. Am J Respir Crit Care Med. 2017;195(2): e4-e19.

6) Akerlund A, Andersson M, Leflein J, et al. Clinical trial design, nasal allergen challenge models, and considerations of relevance to pediatrics, nasal polyposis, and different classes of medication. J Allergy Clin Immunol 2005; 115:S460-82

7) Djukanović R, Feather I, Gratziou C, et al. Effect of natural allergen exposure during the grass pollen season on airways inflammatory cells and asthma symptoms. Thorax $1996,51: 575-581$

8) de Kluijver J, Evertse CE, Schrumpf JA, et al. Asymptomatic worsening of airway inflammation during low-dose allergen exposure in asthma: protection by inhaled steroids. Am J Respir Crit Care Med 2002, 166:294-300

9) Liu LY, Swenson CA, Kelly EA, et al. Comparison of the effects of repetitive low-dose and single-dose antigen challenge on airway inflammation. J Allergy Clin Immunol $2003,111: 818-82$ 
10) Takeda K, Gelfand EW. Mouse models of allergic diseases. Curr Opin Immunol. 2009 Dec;21(6):660-5

11) Bousquet J, Khaltaev N, Cruz AA, et al. Allergic Rhinitis and its Impact on Asthma (ARIA) 2008 update (in collaboration with the World Health Organization, GA(2)LEN and AllerGen). Allergy 2008;63 (Suppl 86):8-160.

12) Eifan AO, Orban NT, Jacobson MR, Durham SR. Severe persistent allergic rhinitis: inflammation but no histologic features of structural upper airway remodeling. Am J Respir Crit Care Med 2015;192(12):1431-9.

13) Varney VA, Jacobson MR, Sudderick RM, et al. Immunohistology of the nasal mucosa following allergen-induced rhinitis. Identification of activated $\mathrm{T}$ lymphocytes, eosinophils, and neutrophils. Am Rev Respir Dis. 1992 Jul;146(1):170-6

14) Sullivan P, Stephens D, Ansari T, et al. Variation in the measurements of basement membrane thickness and inflammatory cell number in bronchial biopsies. Eur Respir J 1998;12:811-815.

15) Nicholson GC, Kariyawasam HH, Tan AJ, et al. The effects of an anti-IL-13 mAb on cytokine levels and nasal symptoms following nasal allergen challenge. J Allergy Clin Immunol. 2011 Oct;128(4):800-807

16) Leaker BR, Malkov VA, Mogg R, et al. The nasal mucosal late allergic reaction to grass pollen involves type 2 inflammation (IL-5 and IL-13), the inflammasome (IL-1 $\beta$ ), and complement. Mucosal Immunol. 2017 Mar;10(2):408-420.

17) Korsgren M, Andersson M, Borgå O, et al. Clinical efficacy and pharmacokinetic profiles of intranasal and oral cetirizine in a repeated allergen challenge model of allergic rhinitis. Ann Allergy Asthma Immunol. 2007;98:316-321. 
18) Fokkens WJ, Godthelp T, Holm AF, Blom H, Mulder PG, Vroom TM, Rijntjes E. Dynamics of mast cells in the nasal mucosa of patients with allergic rhinitis and nonallergic controls: a biopsy study. Clin Exp Allergy. 1992, 22, 701-10

19) Rousseau MC, Boulay ME, Goronfolah L, et al. Comparative responses to nasal allergen challenge in allergic rhinitic subjects with or without asthma. Allergy Asthma Clin Immunol. 2011 Apr 20;7(1):8.

20) Andersson M, Rimmer J, Salome C, et al. Dual symptomatic and exudative nasal responses are not characteristics of perennial allergic rhinitis. Acta Otolaryngol $2001 ; 121: 407-13$

21) Gronborg H, Bisgaard H, Romeling F, Mygind N. Early and late nasal symptom response to allergen challenge: the effect of pretreatment with a glucocorticosteroid spray. Allergy 1993;48:87-93.

22) Banfield G, Watanabe H, Scadding G, et al. CC chemokine receptor 4 (CCR4) in human allergen-induced late nasal responses. Allergy. 2010 Sep;65(9):1126-33.

23) Diamant Z, Gauvreau GM, Cockcroft DW, et al. Inhaled allergen bronchoprovocation tests. J. AllergyClin. Immunol. 132, 1045-1055.

24) McDermott RA, Nelson HS, Dreskin SC. Mediator measurements after daily instillation of allergen: Increased IL-5 and decreased IFN-gamma. Allergy Asthma Proc 2008, 29:146-151.

25) Wachs M, Proud D, Lichtenstein LM, et al. Observations on the pathogenesis of nasal priming. J Allergy Clin Immunol 1989; 84:492-501.

26) Baroody FM, Shenaq D, DeTineo M, et al. Fluticasone furoate nasal spray reduces the nasalocular reflex: a mechanism for the efficacy of topical steroids in controlling allergic eye symptoms. J Allergy Clin Immunol 2009; 123:1342-8. 
27) Eifan AO, Durham SR. Pathogenesis of rhinitis. Clin Exp Allergy. 2016 Sep;46(9):1139-51.

28) Baroody FM, Brown D, Gavanescu L, et al. Oxymetazoline adds to the effectiveness of fluticasone furoate in the treatment of perennial allergic rhinitis. J Allergy Clin Immunol. 2011 Apr;127(4):927-34.

29) Masuyama K, Till SJ, Jacobson MR, et al. Nasal eosinophilia and IL-5 mRNA expression in seasonal allergic rhinitis induced by natural allergen exposure: effect of topical corticosteroids. J Allergy Clin Immunol. 1998 Oct;102(4 Pt 1):610-7.

30) Grainge CL, Lau LC, Ward JA, et al. Effect of bronchoconstriction on airway remodeling in asthma. N Engl J Med 2011;364:2006-2015

31) Kariyawasam HH, Aizen M, Barkans J, et al. Remodeling and Airway Hyperresponsiveness but Not Cellular Inflammation Persist after Allergen Challenge in Asthma. Am J Respir Crit Care Med. 2007 May 1;175(9):896-904.

32) Pegorier S, Wagner LA, Gleich GJ, Pretolani M. Eosinophil-derived cationic proteins activate the synthesis of remodeling factors by airway epithelial cells. J Immunol 2006;177:4861-4869

33) Koya T, Kodama T, Takeda K, et al. Importance of myeloid dendritic cells in persistent airway disease after repeated allergen exposure. Am J Respir Crit Care Med. 2006 Jan $1 ; 173(1): 42-55$.

34) Lim YS, Won TB, Shim WS, et al. Induction of airway remodeling of nasal mucosa by repetitive allergen challenge in a murine model of allergic rhinitis. Ann Allergy Asthma Immunol. 2007 Jan;98(1):22-31.

35) Lee JJ, Dimina D, Macias MP, et al. Defining a link with asthma in mice congenitally deficient in eosinophils. Science 2004;305:1773-1776. 
36) Humbles AA, Lloyd CM, McMillan SJ, et al. A critical role for eosinophils in allergic airways remodeling. Science 2004;305:1776-1779.

37) Fattouh R, Al-Garawi A, Fattouh M, et al. Eosinophils are dispensable for allergic remodeling and immunity in a model of house dust mite-induced airway disease. Am J Respir Crit Care Med. 2011 Jan 15;183(2):179-88.

38) Jacobsen EA, Ochkur SI, Doyle AD, et al. Lung pathologies in a chronic inflammation mouse model are independent of eosinophil degranulation. Am J Respir Crit Care Med. 2017 May 15;195(10):1321-1332.

39) Takeda K, Shiraishi Y, Ashino S, et al. Eosinophils contribute to the resolution of lungallergic responses following repeated allergen challenge. J Allergy Clin Immunol. 2015 Feb;135(2):451-60.

40) Fajt ML, Wenzel SE. Asthma phenotypes and the use of biologic medications in asthma and allergic disease: the next steps toward personalized care. J Allergy Clin Immunol 2015; 135: 299-310.

41) Nguyen TH, Casale TB. Immune modulation for treatment of allergic disease. Immunolo Rev, 2011 Vol. 242: 258-271.

42) Ray A, Raundhal M, Oriss TB, et al. Current concepts of severe asthma. J Clin Invest. 2016;126(7):2394-2403. 
530 Figure 1: Flow chart of study design. Definition of abbreviations: TNSS= Total nasal symptom score. Dil 1-5: Diluent challenges-dose 1 to 5. Grass 1-5: Timothy Grass allergen challengesDose 1 to 5; $A R$ : Seasonal Allergic Rhinitis; $H C$ : Healthy controls; V1-Recruitment visit, V2Repetitive diluent challenge visit; V3- Repetitive allergen challenge.

Figure 2: A) Early phase response (EPR) area under the curve (AUC) post-repetitive allergen or diluent challenge in allergic rhinitis. D1-5: Diluent doses; A1-5: Allergen challenge doses; $N D$ : Normal healthy controls single diluent challenge, $N A$; Normal healthy controls single allergen challenge. B) Late phase response (LPR)-AUC post-repetitive allergen or diluent challenge in allergic rhinitis. D1-5: Diluent doses; A1-5: Allergen challenge doses; $N D$ : Normal healthy controls single diluent challenge, $N A$; Normal healthy controls single allergen challenge. C) Significant correlation between mast cells in nasal submucosa and early phase response (total nasal symptoms scores-AUC) after 1st dose of allergen challenge. D) Immunoreactivity and localization of mast cells stained using Tryptase antibody.

Figure 3: A) Total eosinophil counts in nasal tissue post repetitive allergen challenge compared to diluent for both allergic rhinitis and healthy controls. B) Th2 cytokine (IL-5) levels from nasal fluid post repetitive allergen challenge compared to diluent for both allergic rhinitis and healthy controls. C) Correlation between IL-5 levels from nasal fluid and nasal tissue epithelial eosinophils post $5^{\text {th }}$ dose of repetitive allergen challenge. D) Immunoreactivity and localization of eosinophils stained using major basic protein antibody (MBP). E) Panel E show the nasal tissue eosinophils in an allergic rhinitis subject who underwent repetitive diluent and allergen challenges and biopsies taken at day 1 and day 11 for both post diluent and allergen challenges. Biopsy specimens were immunostained with MBP. 


\begin{tabular}{|c|c|c|}
\hline & $\begin{array}{c}A R \\
(n=12)\end{array}$ & $\begin{array}{l}\text { Normal controls } \\
\qquad(n=16)\end{array}$ \\
\hline Age yrs (Median-Range) & $27(23: 32)$ & $29(24: 42)$ \\
\hline Gender F/M & $4: 8$ & $10: 6$ \\
\hline TNSS **** & $7(4-9)$ & 0 \\
\hline 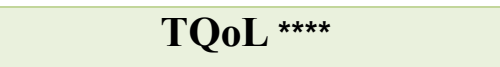 & $2(1.2-3)$ & 0 \\
\hline Eczema \% & 8 & 0 \\
\hline Asthma \% & 16 & 0 \\
\hline Timothy SPT $/ \mathrm{mm}^{2}{ }^{\star \star \star \star}$ & $53(33-70)$ & 0 \\
\hline Specific IgE grass IU/L ${ }^{\star \star \star \star}$ & $17(7-44)$ & 0 \\
\hline Total IgE IU/L ** & $78(34: 175)$ & $11(5.5: 51)$ \\
\hline
\end{tabular}

554

555

$P$ values: ${ }^{* * * *}:<0.0001,{ }^{* *}:<0.01$

556

(median range): $25-75$ centiles

557

558

559

560

561 
Table 2: Nasal tissue, secretion inflammatory cells and cytokines, and serum cytokines

\begin{tabular}{|c|c|c|c|c|c|c|c|c|c|}
\hline & \multicolumn{4}{|c|}{ Seasonal allergic rhinitis } & \multicolumn{2}{|c|}{ Normal Healthy control } & \multicolumn{3}{|c|}{$p$ values } \\
\hline & $\begin{array}{l}1^{\text {st }} \text { dose } \\
\text { Diluent }\end{array}$ & $\begin{array}{l}1^{\text {st }} \text { dose } \\
\text { Allergen }\end{array}$ & $\begin{array}{l}5^{\text {th }} \text { dose } \\
\text { Diluent }\end{array}$ & $\begin{array}{l}5^{\text {th }} \text { dose } \\
\text { Allergen }\end{array}$ & $\begin{array}{l}\text { Diluent } \\
\text { Normal }\end{array}$ & $\begin{array}{l}\text { Allergen } \\
\text { Normal }\end{array}$ & $\begin{array}{c}1^{\text {st }} \text { Dose } \\
\text { Diluent v Allergen }\end{array}$ & $\begin{array}{c}5^{\text {st }} \text { Dose } \\
\text { Diluent v Allergen }\end{array}$ & $\begin{array}{l}\text { Healthy controls } \\
\text { Diluent v Allergen }\end{array}$ \\
\hline & \multicolumn{6}{|c|}{ Epithelial Inflammatory cells (medians $25-75$ centile) } & & & \\
\hline Eosinophils & $0(0)$ & $0(0-16)$ & $0(0)$ & $10(0-157)$ & $0(0)$ & $0(0)$ & 0.5 & 0.06 & 0.5 \\
\hline \multirow[t]{2}{*}{ Mast cells } & $0(0-3)$ & $0(0-3.8)$ & $0(0-8.3)$ & $0(0)$ & $0(0)$ & $0(0-9)$ & 0.63 & 0.5 & 0.06 \\
\hline & \multicolumn{6}{|c|}{ Submucosal Inflammatory cells (medians $25-75$ centile) } & & & \\
\hline Eosinophils & $0(0)$ & $53(12-150)$ & $0(0)$ & $20(0-85)$ & $0(0-0.6)$ & $0(0-0.6)$ & $<0.01$ & 0.03 & 0.81 \\
\hline Mast cells & $29(2-50)$ & $32(13-38)$ & $12(4-38)$ & $9(0-37)$ & $24(8-45)$ & $23(14-41)$ & 1.00 & 0.57 & 0.61 \\
\hline \multirow[t]{2}{*}{ Basophils } & 0 & $0(0-8)$ & 0 & $0(0-4)$ & 0 & 0 & 0.12 & 0.12 & 0.8 \\
\hline & \multicolumn{6}{|c|}{ Total epithelial and submucosal Inflammatory cells (medians $25-75$ centile) } & & & \\
\hline Eosinophils & $0(0-9)$ & $53(12-166)$ & $0(0-2.8)$ & $32(5-292)$ & $0(0-1)$ & $0(0-0.6)$ & 0.01 & $<0.01$ & 0.57 \\
\hline \multirow[t]{2}{*}{ Mast cells } & $28.5(5-50)$ & 34 (13-49) & $12.6(4-51)$ & $9.5(0-46)$ & $24(8.5-45)$ & $35(23-48)$ & 0.91 & 0.57 & 0.20 \\
\hline & \multicolumn{6}{|c|}{ Nasal secretion cytokines (medians $25-75$ centile) } & & & \\
\hline IL-4 & $0.4(0.2-1)$ & $0.5(0.3-1.5)$ & $0.6(0.1-1.1)$ & $0.8(0.3-2)$ & $0.6(0.3-0.8)$ & $0.4(0.3-0.6)$ & 0.9 & 0.4 & 0.4 \\
\hline IL-5 & $4.5(1.5-8)$ & $6(3-16)$ & $6(1.5-8)$ & $9.6(5.7-34)$ & $3.7(2.4-6)$ & $4.3(3-5.2)$ & 0.4 & 0.01 & 0.7 \\
\hline IFN- $\nu$ & $3(1-4)$ & $2(1-5)$ & $3(1-4)$ & $2.5(1-3)$ & $4.6(2.6-7)$ & $4.8(3-6)$ & 0.85 & 0.38 & 0.78 \\
\hline \multirow[t]{2}{*}{ IL-10 } & $3.5(1.8-13)$ & $3.7(2.3-12)$ & $6.8(1.8-20)$ & 7.1(1.7-16) & $7.8(4.8-12)$ & $11(8-18)$ & 0.49 & 0.7 & 0.07 \\
\hline & \multicolumn{6}{|c|}{ Serum secretion cytokines (medians $25-75$ centile) } & & & \\
\hline IL-4 & $12(1-54)$ & $28(1-137)$ & $20(1-106)$ & $26(2-132)$ & $14(0-72)$ & $4.5(1-33)$ & 0.7 & 0.2 & 0.04 \\
\hline IL-5 & $8(0-81)$ & $12(0-210)$ & $7(1-88)$ & $9(1-183)$ & $5(0-111)$ & $4(0-43)$ & 0.4 & 1.0 & 0.2 \\
\hline IFN- $\boldsymbol{\gamma}$ & $50(4-322)$ & $97(4-921)$ & $61(2-481)$ & $70(4-854)$ & $40(1-449)$ & $22(0-154)$ & 0.31 & 0.72 & 0.76 \\
\hline IL-10 & $15(2-113)$ & $21(2-334)$ & $14(2-195)$ & $16(2-255)$ & $14(3-141)$ & $12(2-61)$ & 0.67 & 0.57 & 0.31 \\
\hline
\end{tabular}



allergen challenge.

\begin{tabular}{|c|c|c|c|c|c|}
\hline \multirow[t]{3}{*}{ Measurement } & \multicolumn{2}{|c|}{ 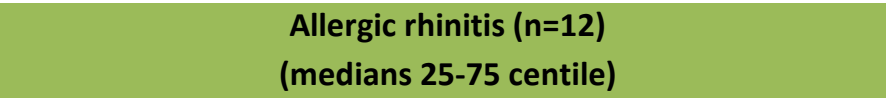 } & \multirow{3}{*}{$\begin{array}{l}\text { Healthy controls }(n=16) \\
\text { (medians } 25-75 \text { centile) } \\
\text { Delta Change Healthy }\end{array}$} & \multicolumn{2}{|c|}{ p-Values } \\
\hline & \multirow{2}{*}{$\begin{array}{l}\text { Delta change Day } 1 \text { NAC } \\
\text { (After } 1^{\text {st }} \text { dose of NAC) }\end{array}$} & \multirow{2}{*}{$\begin{array}{l}\text { Delta Change Day11 NAC } \\
\text { (After 5th dose of RAC) }\end{array}$} & & & \\
\hline & & & & $\begin{array}{c}\text { Day } 1 \text { NAC } \\
\text { AR vs HC }\end{array}$ & $\begin{array}{c}\text { Day } 11 \\
\text { AR vs HC }\end{array}$ \\
\hline Epithelial Integrity & $-1.0(-1.87$ to 0.5$)$ & 0 (-1.0 to 0.37$)$ & $0(-0.5$ to 1.0$)$ & 0.07 & 0.35 \\
\hline $\begin{array}{l}\text { Total Glandular (Epithelial \& } \\
\text { Submucosal glands) \% Area }\end{array}$ & $-11.1(-20.4$ to 17.8$)$ & $13.88(-32.9$ to 23.53$)$ & $-3(-8.5$ to 7.4$)$ & 0.48 & 0.45 \\
\hline RBM Thickness, $\mu \mathrm{m}$ & $-1.4(-4.6$ to 0.74$)$ & $-2.1(-3.3$ to 2.75$)$ & $0.4(-1.58$ to 2.29$)$ & 0.06 & 0.26 \\
\hline CD31 ${ }^{+}$Cells $/ \mathrm{mm}^{2}$ & $19.3(-75.9$ to 203.5$)$ & $-32.8(-207.7$ to 164.1$)$ & 31.78 (-78.8 to 233.9$)$ & 1.0 & 0.37 \\
\hline CD31+ Blood vessels $/ \mathrm{mm}^{2}$ & $-4.36(-31.1$ to 23$)$ & $0(-23.1$ to 25.4$)$ & $2.1(-10.7$ to 25$)$ & 0.55 & 0.64 \\
\hline $\begin{array}{c}\text { CD31 }{ }^{+} \text {Blood vessels } \\
\% \text { vascular area }\end{array}$ & 0 (-1.5 to 0.8$)$ & $-0.8(-3.7$ to 0.3$)$ & $-0.8(-1.7$ to 1.4$)$ & 0.79 & 0.41 \\
\hline Mean size blood vessels ( $\mu \mathrm{m} 2)$ & $-175(-772$ to 249$)$ & $-350(-1685$ to 850$)$ & $-310(-695$ to 289$)$ & 0.81 & 0.56 \\
\hline D2-40+ Cells $\left(\mathrm{mm}^{2}\right)$ & $0(-8.2$ to 6.6$)$ & $0(-1.36$ to 36.4$)$ & $0(-2.5$ to 27.8$)$ & 0.55 & 0.92 \\
\hline D2-40+ Lymphatic vessels $\left(\mathrm{mm}^{2}\right)$ & 0 (0 to 1.5$)$ & 0 (-0.96 to 2.43$)$ & 0 (-0.8 to 4.7$)$ & 0.89 & 0.68 \\
\hline $\begin{array}{c}\text { D2-40 } \% \text { Lymphatic vascular } \\
\text { area }\end{array}$ & $0(-0.27$ to 0.1$)$ & 0 (0.14 to 0.18$)$ & $0(-0.01$ to 0.1$)$ & 0.64 & 0.92 \\
\hline VEGF-A ${ }^{+}$vessels $\left(\mathrm{mm}^{2}\right)$ & $0.92(-1.5$ to 14.3$)$ & $-0.5(-8.6$ to 11.9$)$ & $6(-4$ to 13.6$)$ & 0.88 & 0.5 \\
\hline MMP-7 & $0(-15$ to 16.6$)$ & $0(-3.6$ to 2.1$)$ & $0(-1.2$ to 14.1$)$ & 0.99 & 0.58 \\
\hline MMP-9 & 7.5 (0 to 38.5 ) & $-0.83(-36.9$ to 10.7$)$ & $-0.2(-3.7$ to 12.5$)$ & 0.20 & 0.59 \\
\hline TIMP-1 & 3.7 (0 to 92.5 ) & $0(-2.0$ to 4.4$)$ & 2.5 (0 to 15.7$)$ & 0.26 & 0.17 \\
\hline
\end{tabular}


